\title{
Scalar mass corrections from compact extra dimensions on the lattice
}

\section{Luigi Del Debbio}

SUPA, School of Physics and Astronomy, University of Edinburgh

Edinburgh EH9 3JZ, UK

E-mail: luigi.del.debbioded.ac.uk

\section{Enrico Rinaldi*}

SUPA, School of Physics and Astronomy, University of Edinburgh

Edinburgh EH9 3JZ, UK

E-mail: e.rinaldi@sms.ed.ac.uk

\begin{abstract}
We explore the phase diagram of the SU(2) Yang-Mills theory in 5 dimensions by numerical simulations. The lattice system shows a dimensionally-reduced phase where the extra dimension is small compared to the four-dimensional correlation length. In this phase, at low energies, this system behaves like a four-dimensional gauge theory coupled to an adjoint scalar field.

By tuning the bare parameters of the lattice model, we identify lines of constant physics, and analyse the behaviour of the adjoint scalar mass as a function of the compactification and the cut-off scales.

The perturbative prediction that the effective theory contains a light particle with a mass that is independent of the cut-off is tested against non-pertubative results.
\end{abstract}

The XXIX International Symposium on Lattice Field Theory - Lattice 2011

July 10-16, 2011

Squaw Valley, Lake Tahoe, California

${ }^{*}$ Speaker. 


\section{Introduction}

Five-dimensional Yang-Mills theories with an extra dimension compactified on a circle predict the existence of a scalar low-energy mode, whose mass renormalisation is suppressed by the remnant of the higher-dimensional gauge symmetry. It is well known that quantum corrections yield divergences in the mass of a scalar particles: the scalar mass receives contributions proportional to the square of the ultra-violet (UV) cut-off. However, the mass of the scalar particle coming from the compactification of a higher-dimensional gauge field remains finite, as suggested by one-loop and two-loop calculations [1].

Since the extra dimensional gauge theory is non-renormalisable, it can only be defined as a regulated theory with an ultra-violet cut-off $\Lambda_{\mathrm{UV}}$. In this theory it is rather surprising to find quantum corrections to the scalar mass independent of $\Lambda_{\mathrm{UV}}$ and taking the form

$$
m_{5}^{2} \equiv \delta m^{2}=\frac{9 g_{5}^{2} N_{c}}{32 \pi^{3} R^{3}} \zeta(3),
$$

where $\zeta$ is the Riemann Zeta-function, $N_{c}$ is the numer of colours, $R$ is the radius of the extra dimension and $g_{5}^{2}$ is the coupling constant of the five-dimensional Yang-Mills theory. However, Eq. (1.1) is valid only in the regime where there is a scale separation between the compactification scale $\Lambda_{\mathrm{R}} \sim R^{-1}$ and the cutoff $\Lambda_{\mathrm{UV}}$, because in this case the details of the regularisation can be neglected.

The aforementioned result makes the compactification mechanism a very interesting and promising scenario to protect the mass of scalar particles from cut-off effects. In this proceeding, we report on our recent work [2] where we studied a simple extra dimensional model (cfr. Sec. 2) regularised on the lattice with periodic boundary conditions. Using numerical simulations in the region of phase space where there is a hierarchy of scales $\Lambda_{U V} \gg \Lambda_{R}$, we are able to study the parametric dependence of the adjoint scalar mass $m_{5}$ on the cut-off $\Lambda_{\mathrm{UV}}$ and on the compactification scale $\Lambda_{\mathrm{R}}$. This allows us to clarify the status of Eq. (1.1) in the non-perturbative regime.

\section{Scales separation in the lattice model}

In recent years, there have been a number of studies on the simplest of these extra dimensional theories on the lattice, namely a SU(2) pure gauge theory on a five-dimensional torus with anisotropic couplings $[3,4,5]$.

We discretise the pure gauge action using the following anisotropic lattice Wilson action:

$$
\mathscr{S}_{W}=\beta_{4} \sum_{x ; 1 \leq \mu<v \leq 4}\left[1-\frac{1}{2} \operatorname{Re} \operatorname{Tr} P_{\mu v}(x)\right]+\beta_{5} \sum_{x ; 1 \leq \mu \leq 4}\left[1-\frac{1}{2} \operatorname{Re} \operatorname{Tr} P_{\mu 5}(x)\right],
$$

where the two bare coupling constants $\beta_{4}$ and $\beta_{5}$ can be tuned independently. This describes a lattice system with two independent lattice spacings $a_{4}$ and $a_{5}$, corresponding respectively to the lattice spacing in the four-dimensional subspace, and in the extra fifth direction; the bigger of the two defines the inverse of the cut-off $\Lambda_{\mathrm{UV}}$.

The anisotropy of the couplings $\gamma=\sqrt{\frac{\beta_{5}}{\beta_{4}}}$ is related to the ratio of the lattice spacings $\xi=a_{4} / a_{5}$. 
At tree-level $\gamma=\xi$, but quantum corrections make $\xi$ deviate from this value. The relation between $\xi$ and $\gamma$ for this action has already been studied in bare parameter space and can be found interpolating the data of Ref. [3]. In the following we restrict ourselves to study $\xi \geq 1$ and the cut-off is given by $\Lambda_{\mathrm{UV}} \sim a_{4}^{-1}$.

Two more parameters in the lattice model can be adjusted in order to realise the desired separation of scales; they are $N_{4}$, the number of lattice sites in the usual four directions, and $N_{5}$, the number of lattice sites in the extra dimension. Together with the corresponding lattice spacings, they determine the physical size of the system: $L_{4}=a_{4} N_{4}$ in four dimensions and $L_{5}=2 \pi R=a_{5} N_{5}$ in the fifth dimension.

Using the model described above, we would like to find a region of its parameters space where we observe the following:

- A separation between the compactification scale and the cut-off

$$
\Lambda_{\mathrm{UV}} \gg \Lambda_{\mathrm{R}}
$$

This translates into the following relation for the lattice model parameters

$$
\frac{a_{5} N_{5}}{a_{4}}=\frac{N_{5}}{\xi} \gg 1
$$

and allows us to rely on the results of the five-dimensional theory when describing the lowenergy physics.

- A separation between the four-dimensional physics and the cut-off

$$
\sqrt{\sigma} \ll \Lambda_{\mathrm{UV}} ; \quad m_{5} \ll \Lambda_{\mathrm{UV}} .
$$

The above relations translate into

$$
a_{4} \sqrt{\sigma} \ll 1 ; \quad a_{4} m_{5} \ll 1 .
$$

If the above relations are true, we expect the long distance physics to be independent of the regularisation details of the theory.

- A separation between the four-dimensional physics and the compactification scale

$$
\sqrt{\sigma} \ll \Lambda_{\mathrm{R}} ; \quad m_{5} \ll \Lambda_{\mathrm{R}} .
$$

In terms of the lattice model we have

$$
\frac{N_{5}}{\xi} a_{4} \sqrt{\sigma} \ll 1 ; \quad \frac{N_{5}}{\xi} a_{4} m_{5} \ll 1 .
$$

If this holds, higher Kaluza-Klein modes do not enter the relevant dynamics for the lowenergy physics.

- A scalar mass $m_{5}$ in physical units independent of the cut-off

$$
\frac{m_{5}^{2}}{\sigma} \propto \Lambda_{\mathrm{R}}^{2}
$$

as expected from Eq. (1.1). 


\section{Phase diagram and results from lattice simulations}

Since it is crucial for our purposes to simulate the theory in the correct phase, we briefly discuss the current understanding of the phase diagram of the $\mathrm{SU}(2)$ pure gauge theory in five dimensions described by the action in Eq. (2.1). Results are available both at $\gamma>1[3,4]$ and $\gamma \leq 1$ [5].

The isotropic model, where the lattice spacings are the same $a_{4}=a_{5}$, has a bulk phase transition when all the dimensions are equal and large. The bulk line separates a confined phase $(\sigma>0)$ that is connected to the strong coupling regime from a Coulomb-like phase $(\sigma=0)$ connected to the weak coupling. This bulk transition disappears when the lattice size in anyone dimension is decreased below a critical size, $L_{c}$, which is the critical length of the Polyakov loop in that direction. Below $L_{c}$ center symmetry is broken. In this case the phase transition becomes a second order one in the same universality class of the four-dimensional Ising model. We take $L_{5}$ to be our compactified length and in Fig. 1 we show the pattern of phase transition for $L_{5}=4 a_{5}$ and $L_{5}=6 a_{5}$ in the region $\gamma>1$. The main feature is that at fixed lattice geometry the nature of the phase transition line strongly depends on the anisotropy: the second order phase transition related to centre breaking merges into the bulk phase transition when $\gamma \lesssim \gamma_{c}$. The emerging physical picture tells us that the disappearance of the bulk phase transition happens as soon as the five-dimensional system compactifies; in other words, $\gamma_{c}$ defines a critical lattice spacing in the extra dimension $a_{5 c}$ that makes $2 \pi R=a_{5} N_{5}$ smaller than the critical $L_{5 c}=a_{5 c} N_{5}$. The interesting region for our purposes, is at $\gamma>\gamma_{c}$ and above the line of second order phase transition, where the extra dimension is smaller than its critical value $L_{5}<L_{5 c}$. We refer to this phase as the dimensionally reduced phase, following Ref. [4].

Since this is the first time that this particular region of the phase space is explored with lattice
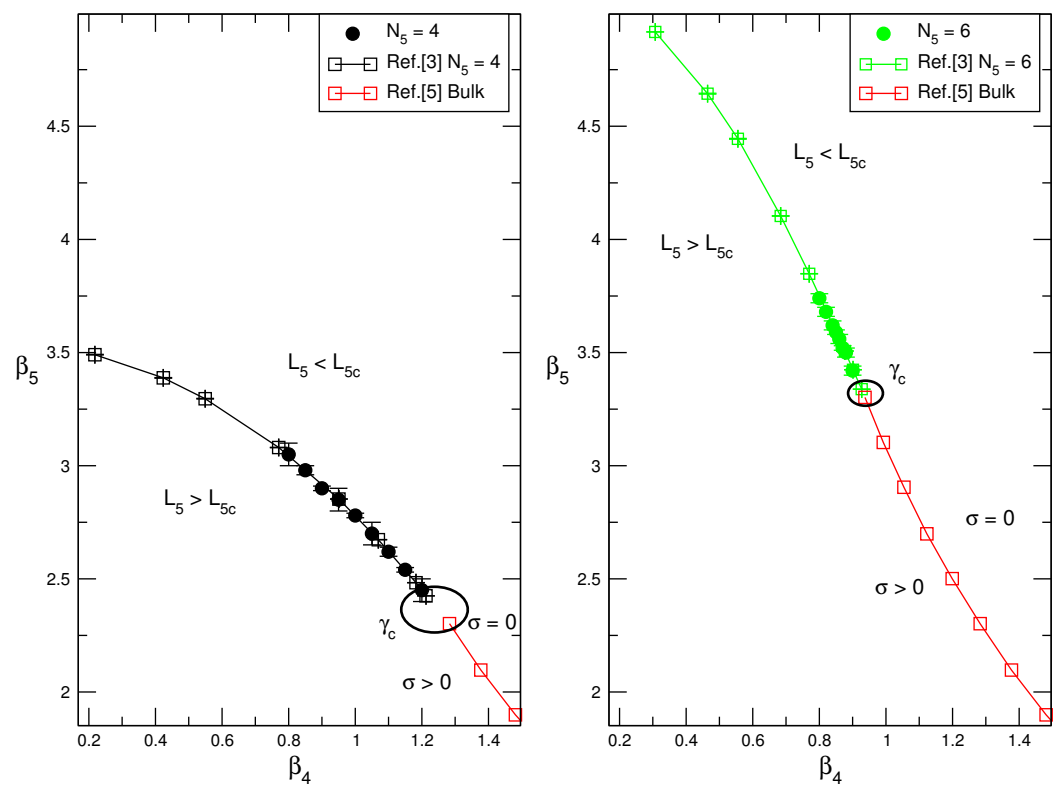

Figure 1: Phase diagram of the five dimensional SU(2) pure gauge lattice model in the $\left(\beta_{4}, \beta_{5}\right)$ plane for different values of $N_{5}$. The bulk phase transition separating a confinement from a Coulomb-like phase disappears for $\gamma>\gamma_{c}$ into a physical thermal-like phase transition. The location of this transition changes in the parameter space as we change $N_{5}$. 

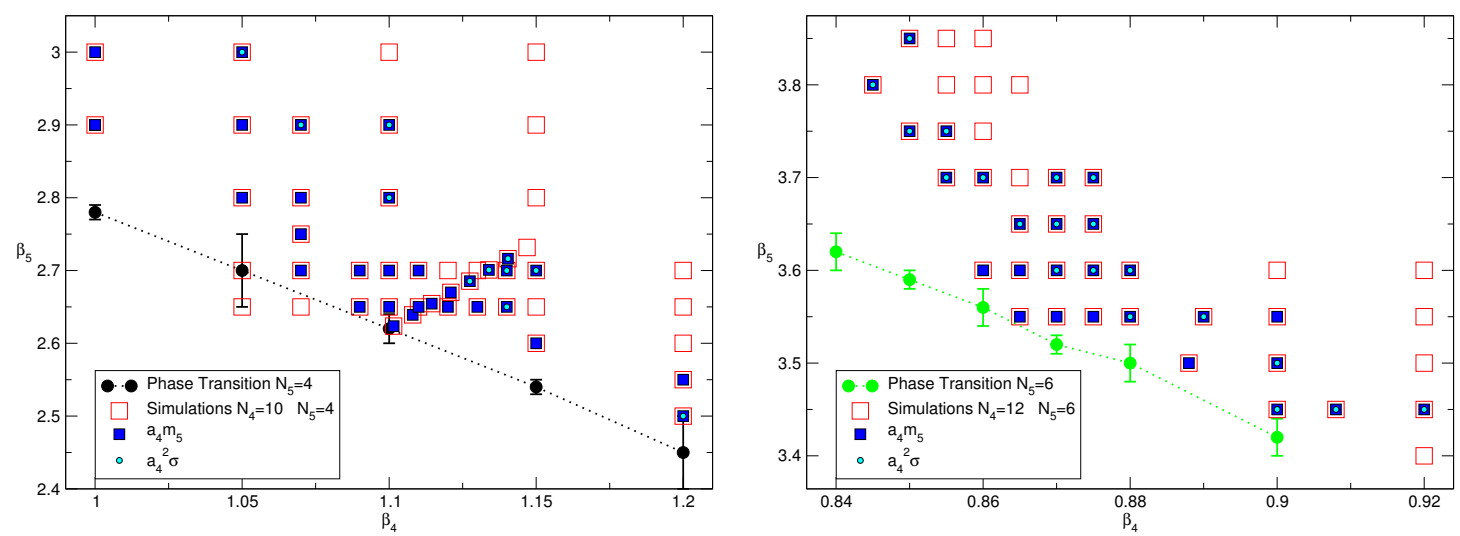

Figure 2: The plots show the region of the phase diagram that we explored with numerical simulations, both for $N_{5}=4$ (left) and $N_{5}=6$ (right). The location of the second order phase transition is also shown. The blue squares are points where the scalar mass $a_{4} m_{5}$ was reliably extracted, whereas the green circles represent points where we were able to measure the string tension $a_{4} \sqrt{\sigma}$.

simulations, we performed a broad scan, aiming primarily at identifying the interesting region. As a consequence, there are lattices for which we were unable to measure precisely both the string tension $a_{4} \sqrt{\sigma}$ and the scalar mass $a_{4} m_{5}$. In Fig. 2, we show all the simulations' points and at the same time we identify the ones where either $a_{4} m_{5}$ or $a_{4} \sqrt{\sigma}$ could not be extracted satisfactorily from correlation functions of suitable gauge invariant operators.

The emergent pattern seems to suggest that the lattice spacing $a_{4}$ changes dramatically in these regions of the phase space. For example, looking at Fig. 2, we notice that the string tension $a_{4} \sqrt{\sigma}$ can only be measured in a small subset of points; the points closer to the line of second order phase transition are characterised by spatial Polyakov loops whose mass is too high for a signal to be extracted reliably. Since the mass of the loops is given by $N_{4} a_{4} \sigma$, in this region the lattice spacing $a_{4}$ is getting larger in units of the string tension. Following our discussion in Sec. 2, we regard the region close to the line of phase transition as the one characterised by a small cut-off $\Lambda_{\mathrm{UV}}$. In this region, there is not a clear separation between the low-energy physics and the cut-off, and we expect to observe large discretisation errors. To make things even more interesting, we find the scalar mass $a_{4} m_{5}$ to be small in this same region, where $a_{4}$ is large. In fact, it turns out to be very difficult to find points in the phase diagram where both $\sqrt{\sigma}$ and $m_{5}$ are separated from the cut-off scale at the same time. This results in a scalar mass $m_{5} \sim \sqrt{\sigma}$ for all the points on which we were able to reliably measure the string tension. On the other hand, a light scalar does exist very close to the second order transition line, where $a_{4} m_{5}$ is small and $a_{4} \sqrt{\sigma}$ is large.

A more quantitative statement can be made by looking at the measured observables as functions of the bare parameters. For example, our data allow us to study the behaviour of $a_{4} \sqrt{\sigma}$ at fixed value of $\beta_{4}$ as we change $\beta_{5}$, and viceversa. The same can be done with $a_{4} m_{5}$. In the left panel of Fig. 3 we see that the scalar mass approaches the cut-off scale $a_{4} m_{5} \gtrsim 1$ as we move away from the line of second order phase transition at fixed $\beta_{4}$. While the scalar mass becomes smaller as we approach the critical line, the opposite happens to the string tension, as it is shown in the right panel of Fig. 3. Each point we have simulated on the phase diagram correspond to a precise location in the space given by the three energy scales $\Lambda_{\mathrm{UV}}, \Lambda_{\mathrm{R}}$ and $m_{5}$. We can therefore translate our results at $N_{5}=4$ 

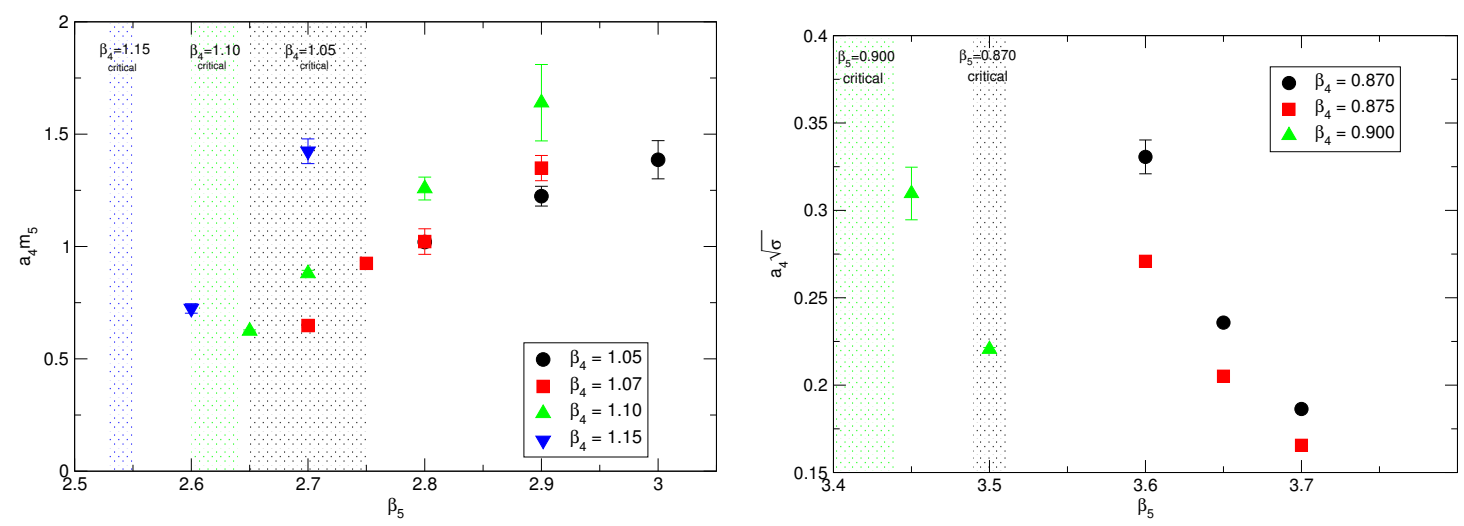

Figure 3: (left) The scalar mass in units of the lattice spacing $a_{4} m_{5}$ as a function of $\beta_{5}$ and for four different values of $\beta_{4}$ at $N_{5}=4$. (right) The string tension in units of the lattice spacing $a_{4} \sqrt{\sigma}$ as a function of $\beta_{5}$ and for three different values of $\beta_{4}$ at $N_{5}=6$. In both panels the approximate location of the critical region is shown.

and $N_{5}=6$ into a common set of points $\left(\Lambda_{\mathrm{UV}}, \Lambda_{\mathrm{R}}, m_{5}\right)$. This approach allows us to study $m_{5}$ as a function of the other two energy scales, instead of the bare parameters. From now on we express the energies $\Lambda_{\mathrm{UV}}$ and $\Lambda_{\mathrm{R}}$ using their length counterpart, $a_{4} \sqrt{\sigma}$ and $R \sqrt{\sigma}$ respectively. This two length scales are related to each other by Eq. (2.3) and they are both measured non-perturbatively: the first is directly measured, whereas the second relies on our interpolation of $\xi$ from Ref. [3]. With our available data, we can explore the behaviour of the scalar mass $m_{5}$ in the following region of lattice spacings $a_{4}$ and compactification radii $R$

$$
0.15<a_{4} \sqrt{\sigma}<0.40, \quad 0.05<R \sqrt{\sigma}<0.12 .
$$

The major advantage of interpreting the data in this new physical space is that we can disentangle compactification effects from cut-off effects. Since our values for the lattice spacing usually correspond to different compactification radii, we look at the combination $m_{5} R$. This is expected to be independent on $R$, while retaining any dependence on the cut-off. If Eq. (1.1) holds, then $m_{5} R$ should be independent of $R$ and $a_{4}$ at leading order. In Fig. 4 we plot $m_{5} / \sqrt{\sigma}$ as a function of $a_{4} \sqrt{\sigma}$ in the left panel, and $m_{5} R$ in the right panel. The observed range for the scalar mass in units of the string tension is

$$
2<m_{5} / \sqrt{\sigma}<10,
$$

whereas the scalar mass in units of the compactification radius is in the range

$$
0.2<m_{5} R<0.5
$$

The latter range is smaller by almost one order of magnitude for same interval of lattice spacings. This evidence support the observation that the dependence on $a_{4} \sqrt{\sigma}$ is mild.

\section{Conclusions}

In this work we presented a non-perturbative study of pure $\mathrm{SU}(2)$ gauge theory in five dimensions. If the scales of the theory are properly separated, we expect the low-energy dynamics of this 
theory to describe a four-dimensional gauge theory coupled to a scalar field. We have measured the mass of the scalar particle in a specific region of the bare parameters space, where we expect to find the desired separation between physical scales. We have also determined numerically the four-dimensional lattice spacing in units of the string tension.

The final picture seems to confirm the possibility of effectively describing a four-dimensional Yang-Mills theory with a scalar adjoint particle in the continuum limit. Even though the search for a light scalar requires fine tuning in this simple model, we have shown that its mass is only very mildly affected by the ultra-violet cut-off, whereas it strongly depends on the radius of the compactified extra dimension. This is entirely compatible with the perturbative result of Eq. (1.1) and it is the first non-perturbative evidence that the mass of scalar particles coming from a compactification mechanism does not have a quadratic dependence on the cut-off.

\section{References}

[1] Y. Hosotani, Dynamical mass generation by compact extra dimensions, Phys. Lett., B126 (1983) 309; H. Cheng, K. Matchev, M. Schmaltz, Radiative corrections to Kaluza-Klein masses, Phys. Rev., D66, (2002) 036005;

L. Del Debbio, E. Kerrane, R. Russo, Mass corrections in string theory and lattice field theory, Phys. Rev., D80, (2009) 025003.

[2] L. Del Debbio, A. Hart, E. Rinaldi, under completion.

[3] S. Ejiri, J. Kubo, M. Murata, A study on the nonperturbative existence of Yang-Mills theories with large extra dimensions, Phys. Rev., D62 (2000) 105025.

[4] P. de Forcrand, A. Kurkela, M. Panero, The phase diagram of Yang-Mills theory with a compact extra dimension, JHEP, 06 (2010) 050.

[5] F. Knechtli, M. Luz, A. Rago, On the phase structure of five-dimensional SU(2) gauge theories with anisotropic couplings, (2011) [arXiv:1110.4210].
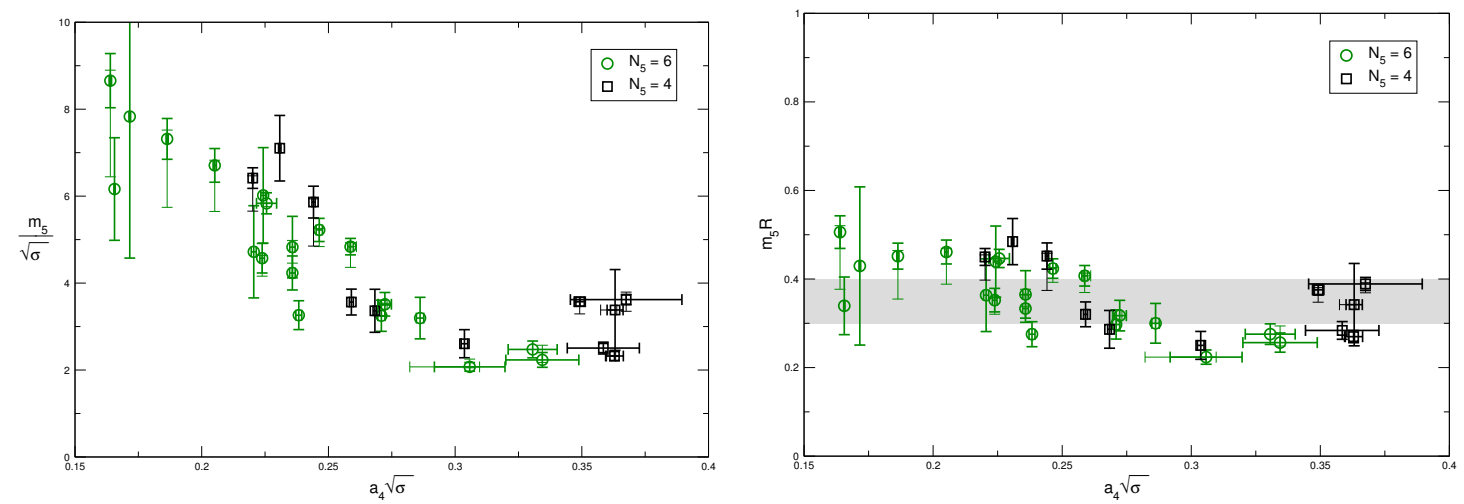

Figure 4: In this plot we show the dependence on the cut-off length given by the lattice spacing $a_{4} \sqrt{\sigma}$ for two observables: (left) the scalar mass in units on the string tension and (right) the scalar mass in units of the compactification radius. The grey band includes all points within two standard deviations. When present, systematic errors are shown with thinner error bars, whereas the thicker ones represent standard deviations. 